\title{
Is this the possible explanation to the unpredictability of the direction of of gamma ray emission during electron-positron annihilation?
}

\author{
Jayaram A S \\ Associate professor, \\ Department of Mechanical engineering \\ Dr Ambedkar Institute of Technology \\ Bangaloe, India \\ June 1, 2021
}

\begin{abstract}
This paper shows the various parameters to be considered, during electron - positron annihilation. Many of the theories suggest that direction of gamma ray emission cannot be predicted. This is normally justified by using quantum mechanics. This paper gives an alternative explanation to unpredictability of gamma ray direction. This explanation does not require the logic of quantum mechanics. It requires modification of classical mechanics to account of the forces between colliding particles. The logic of this paper is that in case of pair production involving gamma ray and electron- positron pair, the classical mechanics calculation itself shows perfect conservation of direction and magnitude of momentum. There, a little recoiling of the nucleus is observed for conserving momentum. Hence it must be possible to use classical mechanics for the process of electron- positron annihilation process also.
\end{abstract}

\section{Introduction}

Let us consider perfect elastic collision of two particles which are of the same mass and same size. let them collide at right angles to each other. Classical mechanics can easily predict the directions of moments after the collision.

The governing equation of momentum is

$$
m 1 u 1+m 2 u 2=m 1 v 1+m 2 v 2 .
$$

here, $\mathrm{m} 1$ and $\mathrm{m} 2$ are the masses of particles, $\mathrm{u} 1$ and $\mathrm{u} 2$ are their respective initial velocities 


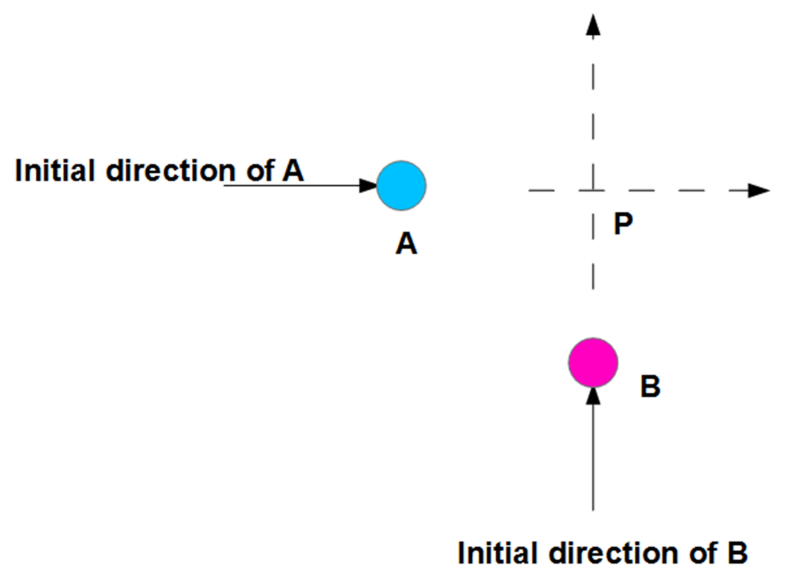

Fig. 1: Two objects colliding at right angles.

$\mathrm{v} 1$ and v2 are their respective final velocities.

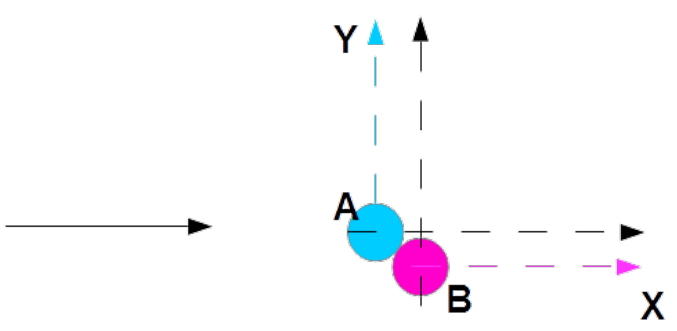

Fig. 2: Final direction of particles.

Since it is assumed as perfectly elastic collision, the initial momentum and the final moment terms are equal. Here, there is a perfect conservation of momentum, both in magnitude and direction. The final direction of A will be along AY and B will be along BY, as shown by dotted lines.

\subsection{Particles with forces}

In case of particles which are having forces between them, like gravitational force, electrostatic force, magnetic force etc, the same old classical method of 
doing the impact and calculating initial and final momentum based on initial directions and velocities will give errors. As a simple example, let us con-

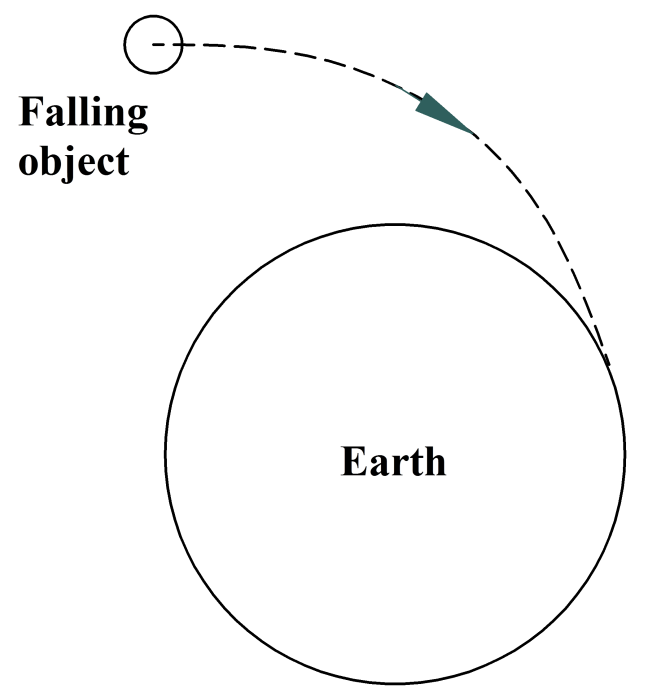

Fig. 3: Object falling towards Earth

sider an object falling under the force of gravity of the Earth. The object will not fall along a vertically straight line but follow a curved trajectory as shown in figure-3. This is mainly because the initial direction of the object will not be exactly vertical. Even if it is having exactly vertical direction of velocity, it will not exactly fall in a straight line because of the effects of atmosphere, gravitational force gradient, Coriolis force and so on. Whenever particles with forces in between them are colliding, then the final direction may be different compared to the initial direction. This is because the forces between the particles will alter the path of movement. This is illustrated as explained below. Let us consider two particles with considerable gravitational force between them are colliding at right angles to each other. From the initial measurements of their masses and gravitational forces, we cannot calculate the final direction of collision. Approximate path is shown in the figure-5. It clearly indicates at the final direction of collisions a totally different compared to the initial directions. The final direction of A will be along AY and B will be along BY, as shown by dotted lines. If we apply simple classical mechanics for the above case, we feel that directional momentum is not conserved. Also, the velocity of particles will gradually increase till they collide, because of gravitational at- 


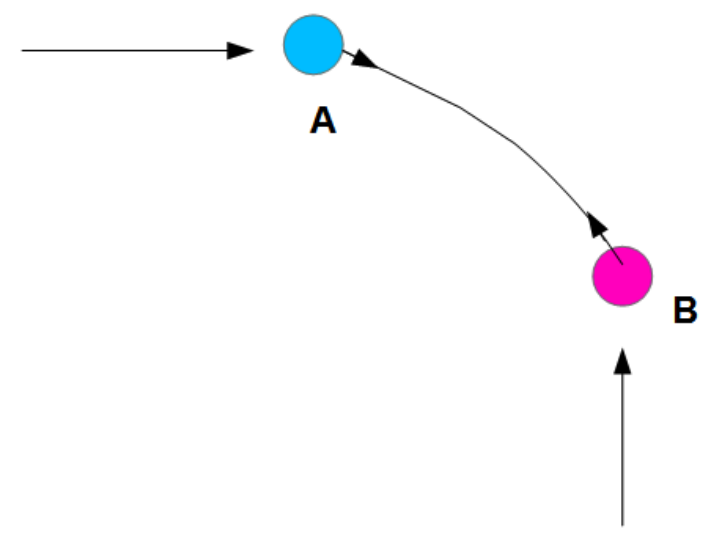

Fig. 4: Change is directions of objects due to force between them.

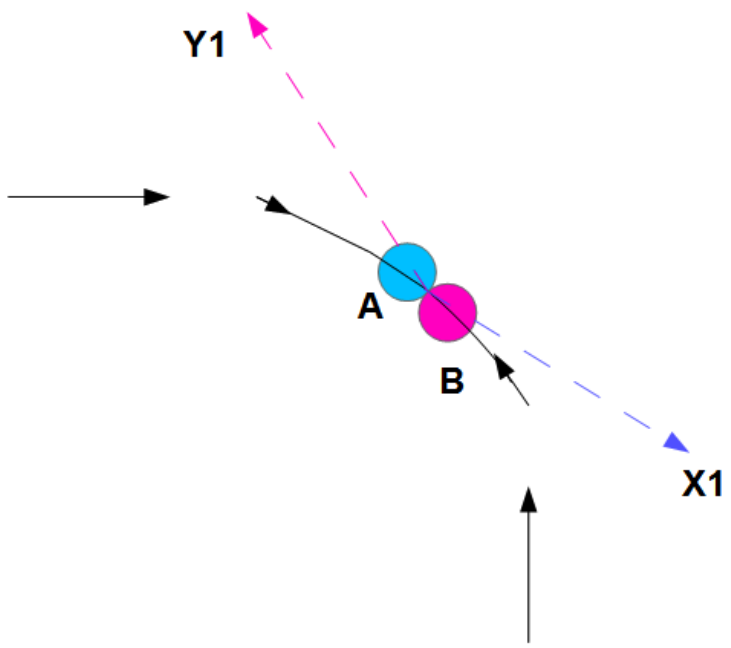

Fig. 5: Colliding position and final directions of movement 
traction. This was the potential energy in the form of gravity, getting converted to kinetic energy. So, at the time of collision, the total momentum will be more than the momentum when the particles started.

\subsection{When the required information cannot be observed}

Let us examine the effect when the observer is obscured from observation during collision, as shown in the figure-6. When the particles just become observ-

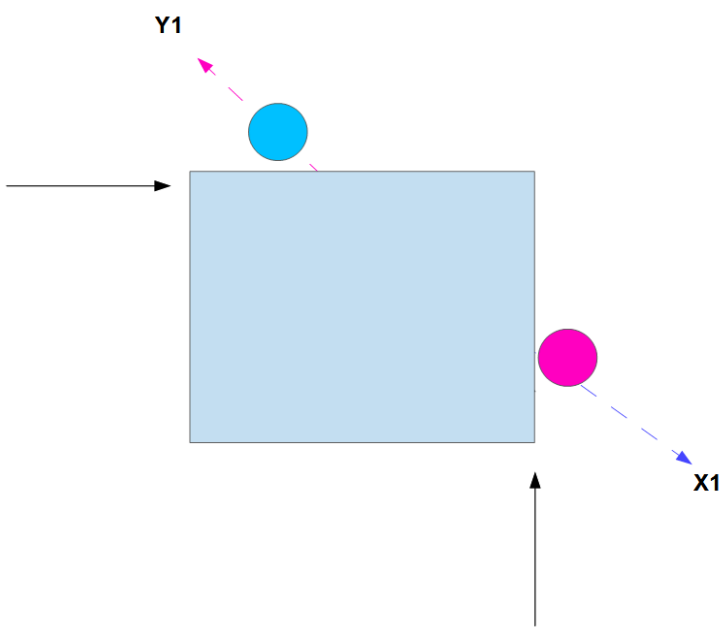

Fig. 6: Missing information required for calculation.

able to him after collision, the observer measures more momentum because of conversion of potential energy to kinetic energy. So, he feels that momentum is not conserved in both magnitude and direction! But after the particles move away through considerable distance, velocity gradually reduces due to gravitational attraction. Then the observer feels that the magnitude of momentum is conserved but not the direction. In this example,the direction is not assumed to change after collision because, almost perfect head-on collision is assumed and so, the movement will be 180 degree from each other.This example shows that unless we precisely know the masses, elasticity, initial velocity and so on, we cannot find out the exact directions of movement of particles after collision. If the particles are very big, having extremely high forces in between them, then they will not separate out after the collision. The whole energy is converted in breaking up the particles in to smaller particles, sound waves travelling inside the solid as elastic vibrations, electromagnetic waves in the form of heat and 
light etc. This will happen especially in case of asteroids and a special term called "accretion" is used to describe such a phenomenon.

\subsection{When the Particles are having polarity}

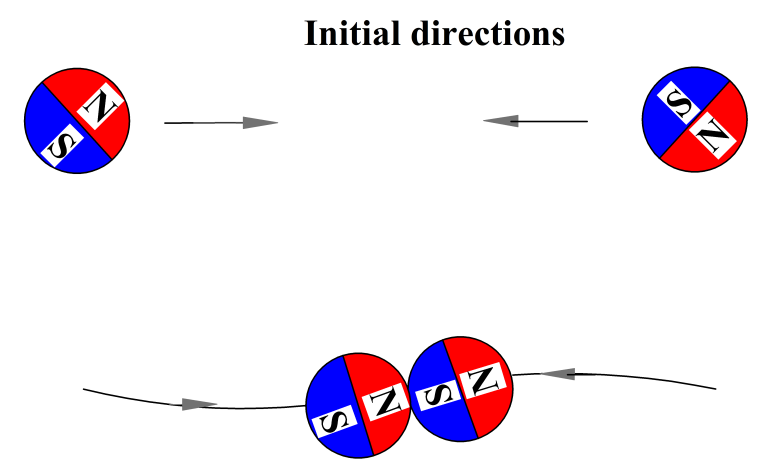

Final direction and orientation

Fig. 7: Initial and final paths of magnets.

The calculation of final direction becomes much more complicated in case when the particles are having polarity. As an example, let us consider two small spherical magnets moving at right angles to each other. Even though there are no big gravitational forces between them, magnetic forces will be there.Depending upon the initial orientation of poles, the final collision will happen after re- orientation of unlike poles getting towards each other. It is shown in the figure-7. It clearly indicates that the gradual deviation of the path of movement of particles with little rotation between the magnets for proper orientation of unlike poles. All these things put together determines the final point of contact and the direction of impact. Here, the sizes and masses of magnets are assumed to be very small, so that the gravitational forces are negligible. Also, magnets will not move away after collision and will not normally break into pieces. The energy will be converted to sound, heat and electromagnetic waves.

\subsection{When the Particles are having electric charges}

Electrons and positrons are having gravity due to mass, polarity due to spin and high attraction force due to opposite charges. All these factors are to be considered to find the final direction and location of impact, which are ex- 
tremely difficult to determine from the initial direction and velocity, even with accurate measurement using cloud chamber[1-3].In some experiments, most of the parameters are considered to some extent[4-5] The force parameters are responsible of what is normally called as hidden momentum. This requires modified equations and rules other than simple classical mechanics[6] but will not require quantum mechanics[7].

In case of electron and positron the opposite charges will make the particles to get attracted and attain very high final velocities. In such cases, relativistic mass equations are also to be considered to calculate the momentum.

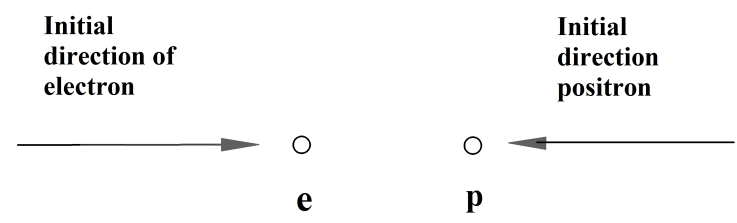

Fig. 8: Initial directions of electron and positron.

They are point particles. Whatever may be the accuracy of measuring instrument, a small error will be there in measuring the initial direction of both the particles. The electron and positron will have initial velocity and spin. So, they will also form a curved path before annihilation, as shown in the figure-9. The electron and positron will have the spin axes. Two electrons will be aligned

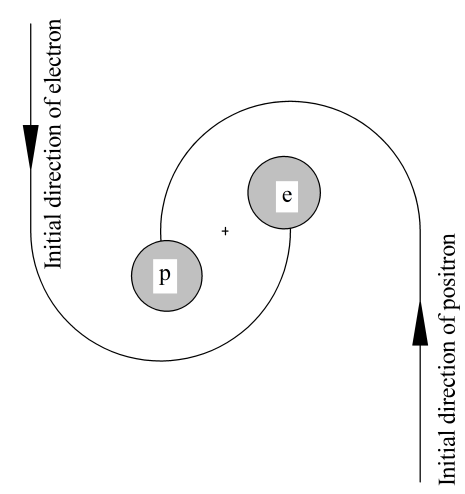

Fig. 9: Re-alignment of electron and positron.

with spin-up and spin-down axes as shown in the figure-10.

But the polarity of positron for the same direction spin of that of that of electron will be opposite, because the charge will be opposite! Hence the alignment of electron and positron will have same spin like spin-up and spin-up, as shown in figure-11. The main thing that we have to notice here is that the alignment 

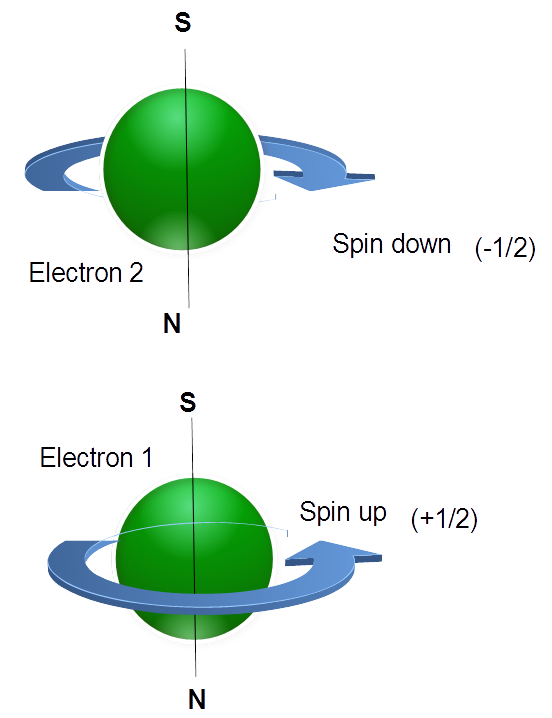

Fig. 10: Alignment of two electrons

S

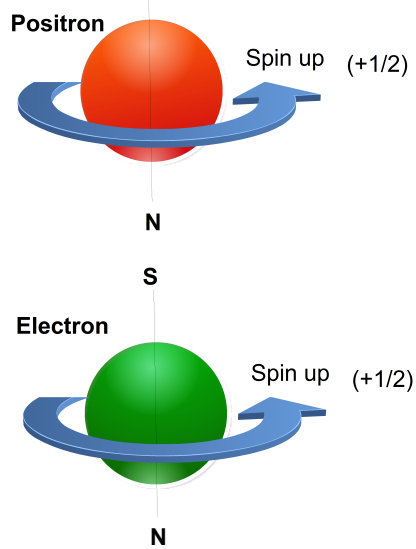

Fig. 11: Alignment of positron and electron

is not just two dimensional. It is actually three dimensional. So, final direction of collision will be normally different from initial direction and the gamma ray will be produced depending upon the final direction of collision, as shown in figure-12. 


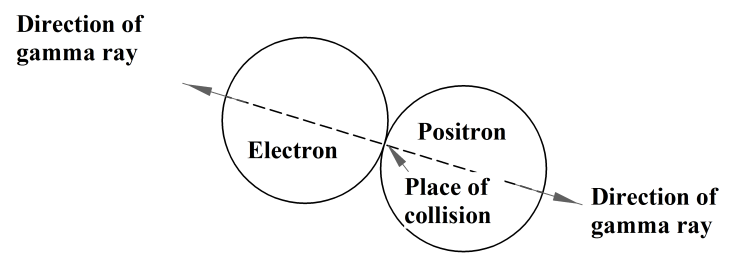

Fig. 12: Final positions of collision and directions of gamma rays

\section{Discussion and conclusion}

- The velocities between electron-positron are very high when they are very near to each other.In such case we have to use relativistic equations for finding momentum.

- Even with accurate measurement using cloud chamber or bubble chamber, the final position can be found out but not the final direction of collision.

- This paper gives an alternative explanation for which quantum mechanics concept is not required.

- This paper shows that if one can measure the final direction of collision, the direction of gamma rays can be predicted

\section{References}

[1] Lee, C. Stationary states of an electron-positron system and annihilation transitions. N. p., 1957. Web.

[2] Teruaki Enoto,Positron Annihilation in Thunderstorms, Nuclear Physics News, 29, no. 3 (2019): 22-27.

[3] Goronwy Tudor Jones., Positron annihilation in flight, Physics Education, 1999,34,5, 276-286.

[4] Arbuzov, Andrej and Bondarenko, Serge and Kalinovskaya, Lidia,Asymmetries in Processes of Electron-Positron Annihilation,Symmetry, volume 12, no 7, 2020:

[5] A Wagner, W Anwand et al,Positron annihilation lifetime spectroscopy at a superconducting electron accelerator, Positron annihilation lifetime spectroscopy at a superconducting electron accelerator, volume 791 (2017):

[6] Jozef Hanca, Slavomir Tulejab,Martina Hancovac,,Symmetries and conservation laws: Consequences of Noether's theorem,American Journal of Physics, Vol. 72, No. 4, pp. 428-435, April 2004.

[7] Basham, C L, Brown, L S, Ellis, S D, and Love, S T. Electron-positron annihilation energy pattern in quantum chromodynamics: Asymptotically free perturbation theory. OSTI.gov: N. p., 1978. Web. doi:10.1103/PhysRevD.17.2298. 